BNL -46672

DE92 001772

\title{
How Robust will the RHIC Lattice be During Commissioning
}

\author{
Sho Ohnuma \\ University of Houston, Texas
}

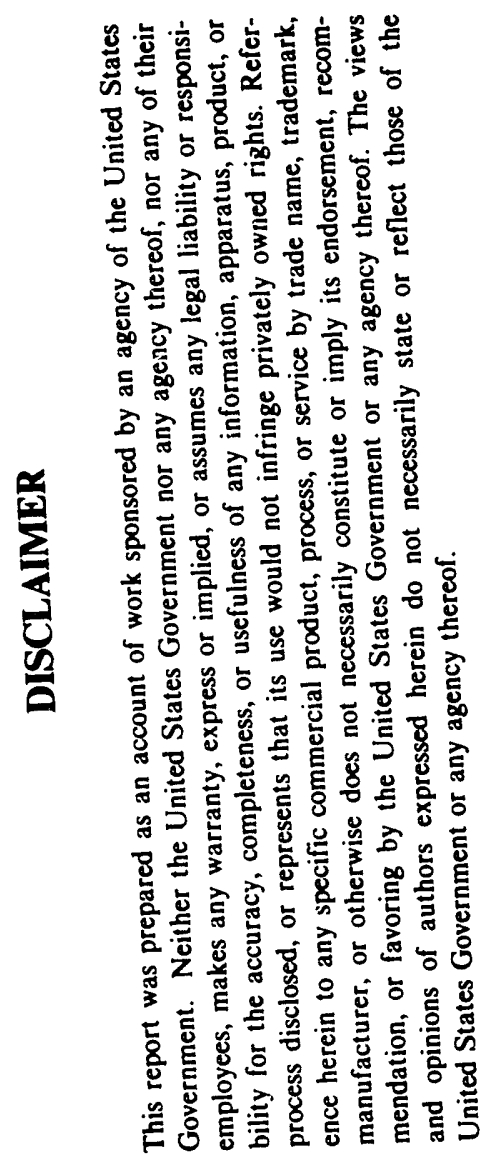

September 1991

\section{R H I C P R O J E C T}

\author{
Brookhaven National Laboratory \\ Associated Universities, Inc. \\ Upton, NY 11973
}




\section{How Robust will the RHIC Lattice be During \\ Commissioning? \\ Sho Ohnuma \\ University of Houston, TX 77204-5508 \\ September 1991

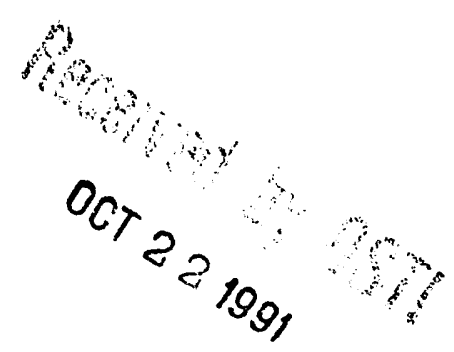

\section{Introduction}

During the workshop on RHIC lattice in August 1991, the review committee expressed its (nearly) unanimous sentiment that the perfect matching of lattice parameters with many independent control variables should not be imposed in the design of insertions. At the same time, the committee recommended that some "knobs" not used during the commissioning stage be kept for possible future needs such as the compensation of detector magnets. Unfortunately, no quantitative guidelines were presented on the degree of allowed mismatch in each lattice parameter except to say that "the matching accuracy required should not be higher than the expected lattice error after RHIC has been fully commissioned and all corrections applied."

A question, which is different from the relaxation of precise matching but definitely related to the commissioning (therefore of more immediate importance), is the expected behavior of the lattice with all control variables set at their nominal design values except for two, that is, the gradient of arc QFs and QDs. In this mode of operation, it is irrelevant to talk about the total number of independent knobs since they are not touched at all.

It is assumed here that the multiturn of the injected beam is already established and the closed orbit is corrected to less than, say, $1 \mathrm{~mm}$ in both horizontal and vertical directions. One may or may not activate the chromaticity-correcting sextupoles at this point but the tune measurement will certainly be at the top of the priority list. For this, it is necessary to have a reliable digitized signal of a kicked beam position (single bunch) lasting for a few hundred turns. Partial activation of the chromaticity sextupoles rnay be necessary if the coherent signal dies down too quickly. 
With the capability of tune measurement at hand, exploration of the tune space to find the optimum working point follows naturally. Since there are any number of insertion quadrupoles which can be controlled independently, it is easy to find the amount of linear coupling by measuring two (normal mode) tunes and their smallest separation. The initial setting of correction skew quadrupoles can be found without too much difficulty by minimizing the split.

The question raised here is whether the RHIC lattice is robust enough to make all these commissioning manipulations possible. There are of course many factors involved in answering this question in a definitive manner. The purpose of this note is to see if there are any fundamental and serious shortcomings basic to the lattice. The lattice considered here is the one presented to the workshop by Steve Tepikian and called RHIC91. More specifically, we fix nine quadrupole parameters in all insertions except in the 6 o'clock insertion where the independent parameters is sixteen. The so-called perfect matching may require fourteen parameters instead of nine but the difference is insignificant. On the other hand, if the number of parameters is reduced from sixteen to nine in the 6 o'clock insertion, the mismatch in the arc beta function becomes non-trivial. For example, the horizontal beta may vary between $40 \mathrm{~m}$ to $60 \mathrm{~m}$ at QF locations.

\section{Ideal Lattice with Perfect Field}

Strictly speaking, the superperiodicity of RHIC91 is one due to the special insertion at 6 o'clock. Thus the intrinsic linear stopband nearest the design operating $\bar{p}$ vint is at $Q=28.5$ with the next two at 28 . and 29 . The one at 28.5 may be troublesome since it is intrinsic for the three-fold symmetry as well. If the width of stopband is too large even for the ideal machine as designed, the tune scanning may encounter some difficulties because of the rapidly increasing values of beta as the tune approaches the stopband.

Following the most likely and practical commissioning procedures, we scan the tune space by using two knobs, one for the arc QFs and the other for QDs but without touching any others. The settings of all insertion quadrupoles are for $\beta^{*}=6 \mathrm{~m}$. 
1.

$$
\begin{aligned}
\left(Q_{x}, Q_{y}\right) & =(28.508,28,517) \\
\beta^{*} & =5.7 \mathrm{~m}-7.0 \mathrm{~m} \\
\max . & =277 \mathrm{~m}
\end{aligned}
$$

$\beta_{x}$ at $\operatorname{arc} \mathrm{QFs}$ or $\beta_{y}$ at $\operatorname{arc} \mathrm{QDs}=35 \mathrm{~m}-66 \mathrm{~m}$

$X_{p}$ at $\operatorname{arc} \mathrm{QFs}=1.62 \mathrm{~m}-1.66 \mathrm{~m}$

2.

$$
\begin{aligned}
\left(Q_{x}, Q_{y}\right) & =(28.019,28.028) \\
\beta^{*} & =5.8 \mathrm{~m}-6.8 \mathrm{~m} \\
\max . \beta & =224 \mathrm{~m} \\
\beta_{x} \text { at arc QFs or } \beta_{y} \text { at arc QDs } & =45 \mathrm{~m}-54 \mathrm{~m}
\end{aligned}
$$$$
X_{p} \text { at } \operatorname{arc} \mathrm{QFs}=1.48 \mathrm{~m}-1.94 \mathrm{~m}
$$

3.

$$
\begin{aligned}
\left(Q_{x}, Q_{y}\right) & =(28.978,28.980) \\
\beta^{*} & =5.8 \mathrm{~m}-6.3 \mathrm{~m} \\
\max \beta & =241 \mathrm{~m} \\
\beta_{x} \text { at } \operatorname{arc} Q F s \text { or } \beta_{y} \text { at } \operatorname{arc} \mathrm{QDs} & =47 \mathrm{~m}-52 \mathrm{~m}
\end{aligned}
$$$$
X_{p} \text { at } \operatorname{arc} \mathrm{QFs}=1.34 \mathrm{~m}-1.83 \mathrm{~m}
$$

Results given here indicate that the stopband widths will not be large enough to cause any troubles in scanning tunes, at least for the ideal ring as designed. It is of course possible to demonstrate the existence of linear stopband when the tune is too close to any integer or half integer values as should be the case for periodicity one. For example, with $\left(Q_{x}, Q_{y}\right)=(28.99,28.994)$, the maximum beta in the ring is more than $700 \mathrm{~m}$ with a wildly varying dispersion function.

\section{Effects of $b_{1}$, the Normal Quadrupole Component in Dipoles}

Effects of $a_{1}$, the skew quadrupole components in dipoles, have been studied extensively by George Parzen. We will simply assume here that most if not all of these effects are reduced to a harmless level by minimizing the tune split. The remaining linear effects are then the ones caused by $b_{1}$. For the example given below, the rms value of $b_{1}$ is taken to 
be $2.0 \times 10^{-4}$ at $25 \mathrm{~mm}$ (i.c., $b_{1}^{\prime}=2.0$ according to the RHIC convention ), the average is zero, and $b_{1}^{\prime} \mathrm{s}$ in all magnets are confined within the $\pm 3 \sigma$.

Chromaticity correcting sextupoles are fixed at $\left(B^{\prime \prime} \ell / B \rho\right)=0.193 \mathrm{~m}^{-2}$ (next to arc QFs) and $-0.366 \mathrm{~m}^{-2}$ (next to arc QDs) so that the chromaticity is zero for the nominal design value of $\left(Q_{x}, Q_{y}\right)=(28.827,28.823)$ at $(\Delta p / p)=0$, with $\beta^{*}=6 \mathrm{~m}$ at all insertions. ${ }^{*}$

It is of course desirable to sample at least a dozen or so cases of random distribution in $b_{1}$ in order to see the performance of "lucky cases" as well as "unlucky cases"; one exarnple alone may create an overly optimistic or pessirnistic view of the reality. The (partial) justification for showing only one randomly chosen case is that the real RHIC should be better than this example if a sorting (even of a simple-minded one) of magnets based on $b_{1}$ and $a_{1}$ is performed in installing magnets in the ring. The expected effects of such a sorting have been studied by Ohnurna and by Dell.

1. $\left(Q_{x}, Q_{y}\right)=(28.827,28.823)$ at $(\Delta p / p)=0$.

For $(\Delta p / p)=0, \beta^{*}=4.85 \mathrm{~m}-10.9 \mathrm{~m}$

$\beta$ at $\operatorname{arc} Q s=29 m-89 m$ (in the focusing plane)

$X_{p}$ at $\operatorname{arc} Q F s=0.6 \mathrm{~m}-2.9 \mathrm{~m}$

$\max . \beta=328 \mathrm{~m}(380 \mathrm{~m}$ for $\Delta p / p=-0.005)$

\begin{tabular}{|lccc|}
\hline$(\Delta p / p)$ & $Q_{x}$ & $Q_{y}$ & $\max .\left|x_{c o}(\mathrm{~mm})\right|$ in arcs \\
\hline-0.005 & 28.827 & 28.804 & 18.4 \\
-0.0625 & 28.827 & 28.814 & 9.1 \\
+0.0025 & 28.827 & 28.832 & 8.7 \\
+0.005 & 28.827 & 28.840 & 17.1 \\
\hline
\end{tabular}

In spite of the large variation in beta and dispersion in the arc, tunes are rather flat, showing that the correction sextupoles are still effective without retuning. However, a large closed orbit excursion at $(\Delta p / p)= \pm 0.005$ may

* After the work was finished, it was realized that, to be more realistic, one should include the average sextupole component $b_{2}$ in dipoles due to persistent current. The required strength of correction sextupoles will then be larger than the values taken here and the momentum acceptance will be less than what is indicated in this example. 
indicate nontrivial effects arising from higher order multipole components in dipoles and the momentum acceptance may not be as large as $\pm 0.5 \%$.

2. $\left(Q_{x}, Q_{y}\right)=(29.180,28.180)$ at $(\Delta p / p)=0$

This choice may arise if the ring prefers the tune split of unity instead of "equal" tunes. It is assumed that the skew quadrupole correction system is effective for this case also.

For $(\Delta p / p)=0, \beta^{*}=3.7 \mathrm{~m}-9.1 \mathrm{~m}$

$\beta$ at arc $\mathrm{Qs}=33 \mathrm{~m}-80 \mathrm{~m}$ (in the focusing plane)

$X_{p}$ at $\operatorname{arc} \mathrm{QFs}=0.0 \mathrm{~m}-2.1 \mathrm{~m}$

$\max . \beta=345 \mathrm{~m}(410 \mathrm{~m}$ for $\Delta p / p=-0.005)$

\begin{tabular}{|lccc|}
\hline$(\Delta p / p)$ & $Q_{x}$ & $Q_{y}$ & $\max .\left|x_{c o}(\mathrm{~mm})\right|$ in $\operatorname{arcs}$ \\
\hline-0.005 & 29.194 & 28.173 & 18.5 \\
-0.0025 & 29.187 & 28.178 & 9.0 \\
+0.0025 & 29.174 & 28.180 & 8.6 \\
+0.005 & 29.170 & 28.179 & 16.6 \\
\hline
\end{tabular}

Again tunes are flat without any retuning of sextupoles.

3. $\left(Q_{x}, Q_{y}\right)=(29.827,28.823)$ at $(\Delta p / p)=0$

This, together with \#2, illustrates that even the linear behavior of the ring is unpredictable when $b_{1}$ error is present. There is no obvious reason to suspect that this choice of tunes will be worse than \#2.

For $(\Delta p / p)=0, \beta^{*}=3.7 \mathrm{~m}-10.9 \mathrm{~m}$

$\beta$ at arc $Q s=30 \mathrm{~m}-91 \mathrm{~m}$ (in the focusing plane)

$X_{p}$ at $\operatorname{arc} \mathrm{QFs}=0.3 \mathrm{~m}-1.5 \mathrm{~m}$

$\max . \beta=346 \mathrm{~m}(848 \mathrm{~m}$ ! for $\Delta p / p=-0.005)$

\begin{tabular}{|lccc|}
\hline$(\Delta p / p)$ & $Q_{x}$ & $Q_{y}$ & $\max .\left|x_{c o}(\mathrm{~mm})\right|$ in $\operatorname{arcs}$ \\
\hline-0.005 & 29.890 & 28.836 & 36. \\
-0.0025 & 29.847 & 28.828 & 10.3 \\
+0.0025 & 29.820 & 28.818 & 7.0 \\
+0.005 & 29.821 & 28.811 & 12.2 \\
\hline
\end{tabular}




\section{Concluding Remarks}

Although the scope of this study was quite limited, results obtained so far do not show any serious defects of the chosen lattice, RHIC91, during the initial stage of commissioning. The study should be repeated in the same manner, that is, with everything except the gradient of arc QFs and QDs fixed but now taking into account the following items:

1) average $b_{1}$ in dipoles due to the persistent current,

2) random $a_{1}$ with the rms value as specified in the design book, together with the twofamily skew quadrupoles adjusted such that the tune split is minimum,

3) random roll of insertion quadrupoles, again with the adjusted skew quadrupoles as above.

Finally, for the success of the commissioning as well as for the future improvements in performance, one can never overemphasize the importance of measuring all multipole field components in cold, resulting in the complete knowledge of the actual ring in the tunnel. Studying many randomly chosen rings as a potential candidate of the real ring is just a game, after all. Although playing this game may be useful at a certain stage of the design activity, one must eventually deal with the real RHIC. Knowledge of the property of its magnets will be indispensable in understanding the beam behavior and in planning the improvement strategies. 

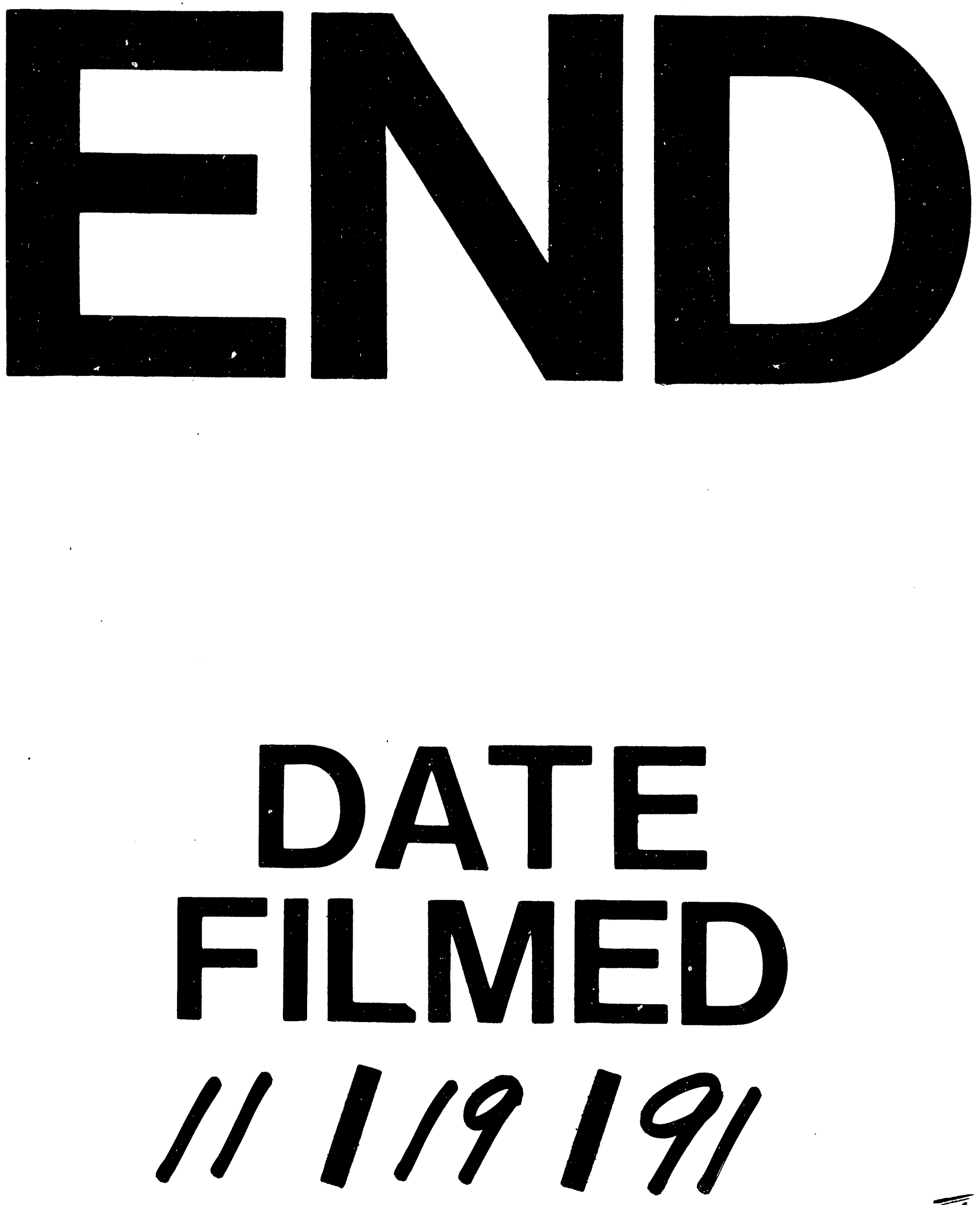

$\bar{I}$ 
